\title{
Responsabilidad Social Universitaria (RSU): Acciones desde la extensión y la proyección social en la Universidad del Magdalena, Colombia, 2012-2016
}

Alexander Daza Corredor* Luis Francisco Miranda TERraza**

Colombia

\section{Resumen}

En lo fundamental, el presente estudio pretende realizar una aproximación sobre las acciones enmarcadas en la extensión y proyección social que ha implementado la Universidad del Magdalena como forma de dar cumplimiento al principio de Responsabilidad Social Universitaria.

\section{Introducción}

Los impactos que generan las universidades pueden ser organizacionales, cognitivos, educativos y sociales (Valleys, 2006). Con relación

* Estudiante Doctorado en Ciencias Gerenciales, Universidad Rafael Belloso Chacín, urbe. Docente de planta de tiempo completo del Programa de Administración de Empresas de la Universidad del Magdalena. Contacto: alexdaza71@hotmail.com

$\because$ Estudiante de grado de Administración de Empresas. Grupo de Investigación en Gestión de las Organizaciones, GIGo. Universidad del Magdalena. Contacto: luisfranc17@gmail.com 
al último aspecto, la extensión social se ofrece como forma de conducir los impactos sociales que genera la universidad, al permitir no solo la interacción universidad-entorno, sino también la intervención por parte de la universidad en el medio que le circunda. Esta es una función misional de las universidades que permite la reciprocidad entre academia y conocimiento y las necesidades de la sociedad.

En este orden de ideas, como principales objetivos de esta investigación se ha planteado, en primer lugar, presentar un marco conceptual sobre la Responsabilidad Social Empresarial (RSU) que proporcione los referentes teóricos para el abordaje de esta temática. En segundo lugar, el estudio presenta los principales hallazgos sobre el avance y estado de desarrollo de la Universidad del Magdalena en materia de extensión y proyección social. Para ello se realizó un estudio descriptivo, centrado en la recolección y análisis documental de información institucional, apoyado en informes realizados sobre los programas y proyectos ejecutados por la Universidad del Magdalena entre el periodo 2012 a 2016, destinados a atender los impactos sociales de la universidad. Con esto se logró hacer una sistematización de las acciones, experiencias e iniciativas encaminadas a la vinculación con la comunidad, que permiten el ejercicio de la responsabilidad social de la universidad.

La investigación da cuenta de la política de extensión y proyección social de la Universidad del Magdalena y su impacto en lo económico, lo político, lo cultural y social tanto en el ámbito local como regional y nacional. Dicha política incluye un sistema de extensión y proyección social que abarca distintas modalidades de intervención que comprende proyectos de innovación científica y tecnológica, consultorías y asesorías, interventorías, servicios de educación, educación continua y permanente, proyectos de creación artística, entre otros servicios de extensión. En ella también se han establecido unos lineamientos que definen las iniciativas estratégicas en materia de extensión en la Universidad del Magdalena.

Así, resulta muy relevante destacar la importancia de la extensión y proyección social de la Universidad del Magdalena, entendida esta función no como un conjunto de actividades filantrópicas o asistencialistas, sino como una vinculación con el entorno social, a una función misional de la universidad con su medio externo. Finalmente, se debe 
señalar que este estudio, además de mostrar un modelo universitario destacado por la innovación en la intervención del entorno social, también constituye un aporte al debate permanente sobre la Responsabilidad Social Universitaria (RSU).

\section{Referentes teóricos}

La sociedad de hoy en día claramente presenta exigencias a todos sus actores, especialmente a las organizaciones que desempeñan un rol importante en los ámbitos económico, social, político y ambiental, razón por la cual deben adaptarse y establecer sus objetivos encaminados a las exigencias de la sociedad. Se entiende por sociedad un grupo de instituciones sociales que, en el marco de su creciente complejidad y diferenciación, define los ámbitos de interacción ciudadana, es decir, todo aquello que haga parte de una expresión social y cultural (Bernal y Rivera, 2011a). Así, desde esta perspectiva, no se debe desconocer que las organizaciones actúan en un marco institucional y regulatorio que determina y forma su comportamiento debido a que las instituciones determinan las reglas de juego en la sociedad (North, 1991; Dacin et al., 2002; Campbell, 2007).

Ahora bien, las organizaciones tienen funciones específicas dentro de la sociedad, por lo tanto desempeñan un rol fundamental en ella. De no existir, la sociedad no estaría perfectamente conformada (Herrera y Jaime, 2004). Dentro de las instituciones educativas, por ejemplo, se destacan las universidades; estas, según Rodríguez (2009), se constituyen en un elemento básico para generar mayores niveles de competitividad en el país y representan una fuente esencial de oportunidades de formación continua y movilidad social. También velan por la calidad y pertinencia de su oferta académica y, como último objetivo, pero no menos importante, se vinculan con su medio aportando al desarrollo de la cultura, las artes y el desarrollo territorial (p. 826). De esta manera, "la universidad hace parte de la sociedad y es considerada como una institución, que cumple objetivos concretos y desempeña funciones en relación con la sociedad general y con cada una de las otras instituciones o componentes que la configuran" (Vallaeys, 
2006, p. 8). Desde sus inicios la universidad surge con la función de vincular sus objetivos con los seres humanos, la ciencia y la sociedad, y se plantean ejes como la enseñanza, la investigación, la extensión o servicio y la promoción de una sociedad democrática (Grondona y Rodríguez, 2000, p. 40).

\section{Responsabilidad Social}

El concepto de responsabilidad social tiene diversas acepciones, y aunque existe una amplia discusión sobre sus orígenes, es con el Global Compact de Naciones Unidas que este término empieza a ser aplicado a ciudadanos, gobiernos, corporaciones, empresas y organizaciones no gubernamentales, entre otros. El Global Compact es una iniciativa "que promueve el compromiso del sector privado, sector público y sociedad civil a alinear sus estrategias y operaciones con diez principios universalmente aceptados en cuatro áreas temáticas: derechos humanos, estándares laborales, medio ambiente y anti-corrupción" (Global Compact, 2016). Estos 10 principios rectores, formulados en 1999, orientan el marco de acción de toda organización, incluyendo a las instituciones de educación superior.

Desde entonces, distintos autores y organismos internacionales han intentado presentar definiciones claras y lo suficientemente categóricas de este concepto, aunque se han dirigido especialmente al ámbito empresarial y de negocios. Sin embargo, es con la Iso 26000 que se logra un marco lo suficientemente amplio para la comprensión de la responsabilidad social que permite su aplicabilidad a todo tipo de organización. Así, según la Guía sobre responsabilidad social de la iso 26000, la responsabilidad social se define como "la responsabilidad de una organización ante los impactos que sus decisiones y actividades ocasionan en la sociedad y en el medio ambiente, mediante un comportamiento ético y transparente" (p. 4).

Es precisamente este enfoque centrado en los impactos el que hace posible abordar el entramado teórico que gira en torno al tema de la responsabilidad social universitaria (RSU). Solo a partir de un cuidadoso análisis desde esta perspectiva es posible descubrir el marco filosófico 
que subyace bajo este complejo concepto, dado que, como expresa Vallaeys (2014), la RSU "no es una mera aplicación a la universidad de los procesos de responsabilidad social empresarial, puesto que los impactos universitarios son genuinos y se cuidan desde las genuinas competencias académicas de la universidad" (p. 108).

\section{Responsabilidad Social Universitaria (RSU)}

A nivel empresarial la tendencia es que las organizaciones implementen con sus stakeholders o grupos de interés, acciones responsables, entendidas estas como "el conjunto de obligaciones y compromisos, legales y éticos, tanto nacionales como internacionales, que se derivan de los impactos que la actividad de las organizaciones producen en el ámbito social, laboral, medioambiental y de los derechos humanos" (Domínguez, 2009, p. 38).

Las universidades, de acuerdo a Vallaeys (2006, p. 4), generan cuatro tipos de impactos (figura 1). El primero de ellos es el impacto de funcionamiento organizacional que se refiere al impacto que genera en la vida del personal administrativo, docente y estudiantil y en el medio ambiente; el impacto educativo que se da de manera directa en la formación de los profesionales ya que a través de este proceso influye en la manera de comportarse y en su rol social; los impactos cognitivos y epistemológicos debido a la orientación que las universidades generan en el saber y las tecnologías y por último, los impactos sociales, ya que estas instituciones se convierten en un referente y en un actor social que puede o no hacer un papel de interlocutor en la sociedad que dé lugar a la solución de problemas 
Figura 1. Impactos de la universidad

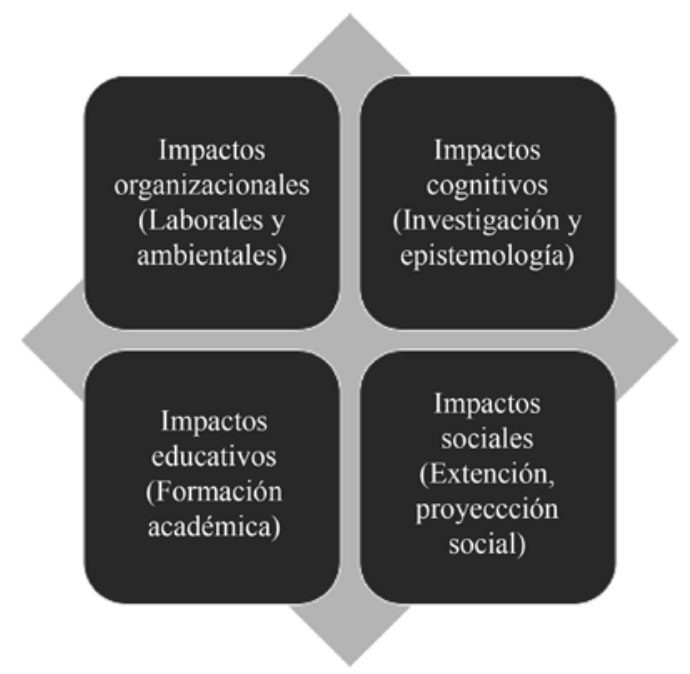

Fuente: adaptado de Vallaeys, De la Cruz y Sasia (2009).

La Responsabilidad Social en las instituciones educativas es aplicable, según Barroso (2009), porque estas contribuyen de manera activa al mejoramiento social, económico y ambiental, a través de la correcta formación de los estudiantes en cuanto a competencias y valores éticos, generación de empleos, relaciones con proveedores y cuidado del entorno que los rodea (p. 52). La responsabilidad social de la universidad como organización implica el cumplimiento de cuatro indicadores esenciales: una buena gobernabilidad, la gestión de los impactos medioambientales y sociales, la rendición de cuentas a las partes interesadas y las alianzas para participar en el desarrollo sostenible (Ojalvo y González, 2014).

Ojalvo y González (2014) también plantean tres ámbitos de acción de la responsabilidad social universitaria. El primero de ellos es el ámbito educativo, el cual se refiere a la formación de estudiantes y profesores, no solamente enfocada a la parte intelectual y técnica sino también a la formación cívica, ética y ciudadana, y en el caso de los docentes se refiere a la formación académica y ciudadana, para que pueda formar al estudiante para la vida, a través de su propio actuar. El segundo ámbito es el del conocimiento, en el cual la universidad a través de la investigación genera y transmite conocimientos científicos 
en pos del progreso social. Por último, aparece un tercer ámbito: el social, que se da a partir del vínculo de la universidad con los actores externos.

La Responsabilidad Social Universitaria, según Vallaeys (2005), citado por Ojalvo y González (2014) es:

una política de calidad ética del desempeño de la comunidad universitaria (estudiantes, docentes y personal administrativo) a través de la gestión responsable de los impactos educativos, cognitivos, laborales, sociales y ambientales que la Universidad genera, en un diálogo participativo con la sociedad para promover el Desarrollo Humano Sostenible (Vallaeys, 2005, p. 8).

Desde otro enfoque, Ruiz (2015) señala que la Responsabilidad Social Empresarial en el caso de las universidades consiste en cumplir a cabalidad su compleja misión institucional, es decir, las tres actividades básicas de una organización académica, como son docencia, investigación y desarrollo institucional, aludiendo que la óptima gestión de estos tres ejes es la clave de su responsabilidad social.

Siguiendo las líneas anteriores, Beltrán, Íñigo y Mata (2014) afirman que la educación superior tiene la responsabilidad social de hacer avanzar la comprensión de problemas con dimensiones sociales, económicas, científicas y culturales, así como la capacidad de hacerles frente. Partiendo de allí se podría decir que la Responsabilidad Social Universitaria (RSU) existe cuando la organización toma conciencia de sí misma, de su entorno y del papel que esta desempeña en dicho entorno. Al respecto, Mendoza-Fernández, Salas-Solano y López-Juvinao (2015) argumentan que a las universidades "les corresponde realizar un autodiagnóstico, primero para involucrar en su pensamiento estratégico la importancia que tiene la responsabilidad social y luego para conocer hasta donde están siendo responsables” (p. 136).

Rodríguez (2010), citado en Caira, Lescher y Lescher (2015), plantea varios modelos de la Rsu, los cuales avanzan de acuerdo con la relación, participación y diálogo de los stakeholders de las universidades, que están conformados por la comunidad universitaria (docentes, estudiantes, personal), el gobierno, la empresa privada, proveedores, 
medios de comunicación, así como las comunidades y organizaciones no gubernamentales. Vallaeys, De la Cruz y Sasia (2009), refiriéndose a los stakeholders, señalan que toda universidad que quiera llevar la RSU a la práctica debe reconocer la legitimidad de las expectativas de los grupos de interés y enfocar su relación con ellos participativa y transparentemente. Conviene pues que cada universidad, desde su propio contexto institucional y social, defina a los grupos de interés externos que quiere atender prioritariamente (p. 63).

Dentro de los modelos propuestos por Rodríguez (2010), se cita el modelo tradicional, el cual enmarca las necesidades sociales-globales en la impartición de conocimientos generales y dentro de este la formación de profesionales; también se encuentra el modelo académico y corporativo, el cual fortalece la docencia y la investigación, orientando a la universidad hacia la satisfacción de intereses específicos. Se habla también del modelo empresarial e instrumental que predica una relación abierta y el enlace funcional entre la universidad y la sociedad, con el diálogo entre la primera y sus respectivas partes interesadas externas e internas, a través de actividades de extensión y, por último, el modelo global, pluralista e intrínseco en el que se crea una estructura organizacional diseñada para la participación de los stakeholders relevantes en la adopción de decisiones en el marco de un gobierno universitario amplio o global.

Después de tener una mejor visión sobre la importancia de la responsabilidad social como marco para la aplicación de políticas, procesos y actividades hacia el medio, se hace necesario indagar a cerca de la aplicación de los proyectos de extensión y proyección social de las universidades, que finalmente terminan siendo una forma de dar cumplimiento a la responsabilidad social universitaria, es decir, la manera que las universidades tienen de proyectarse positivamente ante la comunidad de la cual hacen parte. Inicialmente:

en algunos países la extensión comenzó a ser un componente de la acción política de los estudiantes y de la universidad en la promoción de nuevos modelos de inserción asociados a la lucha política y a las visiones transformadoras, abandonando las lógicas 
reformistas y de transferencia de saberes a las comunidades. (Domínguez, 2012, p. 42)

La extensión y proyección social constituye una función misional de las universidades que permite la reciprocidad entre academia y conocimiento y las necesidades de la sociedad, siendo una función nuclear de las universidades. "La pertinencia, en cuanto al diálogo de la Universidad con su entorno y al cumplimiento de las funciones que le competen, corresponde a una característica propia de la tarea universitaria” (Bernal y Rivera, 2011b, p. 13). Pero la RSU

también abarca la intención de que las actividades y programas de extensión universitaria no sean únicamente un beneficio general para las comunidades, sino que tengan una orientación preferencial hacia las poblaciones que por su vulnerabilidad se encuentran en situaciones de inequidad y marginación social. (Bernal y Rivera, 2011b, p. 13)

\section{Metodología}

En lo fundamental, la presente investigación se aborda como un estudio descriptivo, centrada en la recolección y análisis documental de información institucional apoyada en informes y planes de carácter institucional. "El tratamiento documental significa extracción científico-informativa, una extracción que se propone ser un reflejo objetivo de la fuente original, pero que, soslaya los nuevos mensajes subyacentes en el documento" (Dulzaides y Molina, 2004).

El estudio se realizó en dos fases. En la primera, se realizó una revisión bibliográfica sobre el concepto de responsabilidad social universitaria (RSU) y sobre la extensión y la proyección social en el ámbito universitario en las principales bases de datos académicas. Una segunda etapa comprendió la recolección y análisis de información institucional, apoyados en informes de gestión y en información suministrada directamente por la Vicerrectoría de Extensión y Proyección Social de la Universidad del Magdalena. 
Como pregunta problema para este estudio de corte descriptivo se ha formulado el siguiente interrogante: ¿cuáles son las acciones enmarcadas en la extensión y proyección social que ha implementado la Universidad del Magdalena como forma para dar cumplimiento al principio de Responsabilidad Social Universitaria?

\section{Principales hallazgos o resultados}

\section{Extensión y proyección social en la Universidad del Magdalena}

La Universidad del Magdalena, como institución de educación superior, está llamada a retribuir a la sociedad los impactos causados. En este sentido, es necesario definir las políticas y estrategias que ha implementado este centro de estudios, vinculadas directamente con la proyección y extensión social como forma de dar cumplimiento a su responsabilidad social universitaria.

Para la Universidad del Magdalena la vinculación con el entorno supone la articulación con tres actores fundamentales: el sector productivo, el Estado y la sociedad civil. La interacción con cada uno de estos sectores se da a partir de la identificación de los intereses y necesidades de cada uno, atendiendo a criterios de solidaridad, sostenibilidad y reconocimiento de los ámbitos de acción de cada uno de los actores y su consecuente papel en la construcción del desarrollo, desde la perspectiva de influir de manera positiva en la construcción de políticas públicas conjuntas (Vicerrectoría de Extensión y Proyección Social, 2015).

Mediante estas modalidades de extensión, la Universidad busca mejorar el bienestar de las comunidades, así como el aumento de la productividad y de la competitividad del aparato productivo articulando la docencia, la investigación y la extensión. El objeto de las modalidades de extensión y proyección social es "el intercambio, la aplicación y la integración, en forma dinámica y coordinada, del conocimiento científico, tecnológico, artístico y cultural que se produce en la Universidad, en interacción con el entorno económico, político, cultural y social del país”, bajo los principios de excelencia académica, 
Figura 2. Modalidades de extensión universidad del magdalena

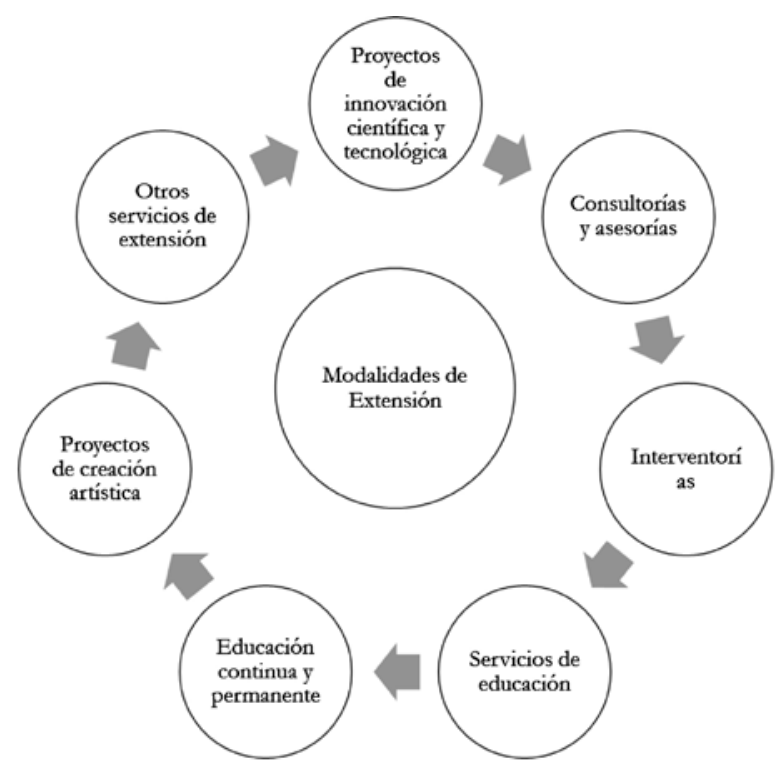

Fuente: elaboración propia a partir de información de página web institucional de la Universidad del Magdalena.

pertinencia, integralidad, articulación, cooperación, reciprocidad, responsabilidad social, ética, sostenibilidad, regionalización (Vicerrectoría de Extensión y Proyección Social, 2015).

\section{Vicerrectoría de Extensión y Proyección Social}

La Universidad del Magdalena, desde la Vicerrectoría de Extensión y Proyección Social (figura 3), gestiona las actividades y proyectos que le permiten acercarse a su entorno y vincularse plenamente con la comunidad, especialmente con las poblaciones más vulnerables (tabla 1). La Universidad del Magdalena tiene presencia en gran parte de la región Caribe de Colombia, contribuyendo mediante la ejecución de proyectos (figura 5) a la población vulnerable de los municipios de la región e incluso con muchos beneficios en todo el país. 
Figura 3. Estructura de la Vicerrectoría de Extensión y Proyección Social

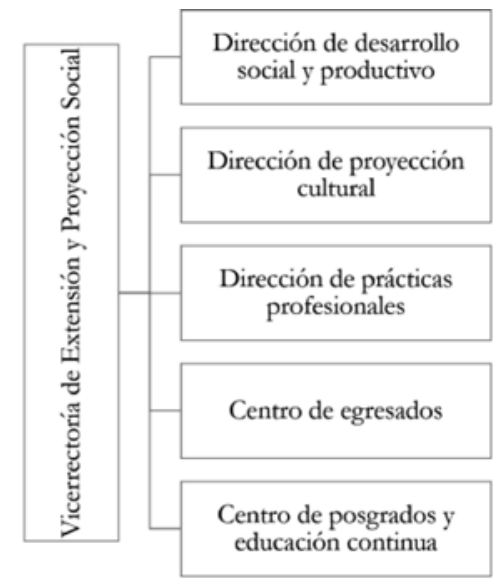

Fuente: elaboración propia a partir de información de página web institucional de la Universidad del Magdalena.

Tabla 1. Población de beneficiados

\begin{tabular}{|l|c|}
\hline \multicolumn{1}{|c|}{ Población de beneficiados } \\
\hline Familias & 190.550 \\
\hline Niños y jóvenes & 60.214 \\
\hline Docentes, padres de familia y otros actores & 19.683 \\
\hline Madres comunitarias, lactantes y/o cabezas de familia & 4.237 \\
\hline Líderes & 1.615 \\
\hline Instituciones educativas & 110 \\
\hline Municipios y comunidades & 195 \\
\hline
\end{tabular}

Fuente: elaboración propia a partir de Informe de Gestión Vicerrectoría de Extensión y Proyección Social 2012-2016.

Gracias al grado de vinculación que ha logrado tener con su entorno, no solo a nivel local sino también a nivel nacional, el Centro Mexicano para la Filantropía (Cemefi) otorgó a la Universidad del Magdalena el Premio a la Mejor Práctica en Responsabilidad Social Empresarial en Latinoamérica, producto de la destacada labor que se realiza desde la Vicerrectoría de Extensión y Proyección Social a través del Programa Escuela de Liderazgo y Formación Ciudadana para el Desarrollo Social. Cemefi anualmente hace reconocimientos a las empresas que cumplan con los estándares establecidos en los ámbitos 
Figura 5. Mapa de presencia institucional de la Universidad del Magdalena

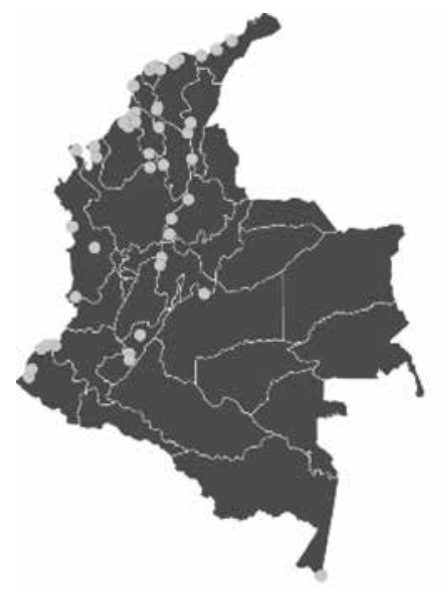

Fuente: tomado de Informe de Gestión Vicerrectoría de Extensión y Proyección Social 20122016.

estratégicos de la responsabilidad social empresarial. Cabe resaltar que las empresas que participan de este proceso lo hacen de manera voluntaria asumiendo públicamente un compromiso con una gestión socialmente responsable y de mejora continua, como parte de su cultura y estrategia de negocio.

El Programa Escuela de Liderazgo y Formación Ciudadana para el Desarrollo Social de la Universidad del Magdalena es

un programa que busca incentivar y fortalecer los procesos de participación democrática en aquellas personas que ejercen el liderazgo en instituciones estatales y comunitarias. Propicia espacios para la concertación y el afianzamiento de la gobernabilidad; fortalecimiento la capacidad de gestión de las comunidades y favoreciendo un trabajo coordinado entre el Estado, la sociedad civil, y las entidades públicas y privadas en pro del desarrollo del Departamento. (Vicerrectoría de Extensión y Proyección Social, 2015)

También a nivel nacional, la Universidad del Magdalena obtuvo el premio "Compromiso con la Responsabilidad Social Empresarial" otorgado por la Federación Nacional de Comerciantes (Fenalco), Capítulo Santa Marta. Fenalco es una entidad de comercio organizado que 
promueve la Responsabilidad Social Empresarial desde el año 1990, cuenta con una alianza con la Corporación Fenalco Solidario Colombia, de la cual hacen parte aquellos empresarios que se comprometen a realizar acciones socialmente responsables. Esta corporación tiene alianzas establecidas con Pacto Global (2010), Global Reporting Initiative (2012), Forum Empresa (2009) y Cemefi (2019), lo que le da un alto nivel de credibilidad a sus procesos y en Colombia se generaron algunas alianzas estratégicas en el año 2015 con organizaciones y proyectos locales, regionales y nacionales: Enplanta y la Universidad Santo Tomás (Corporacion Fenalco Solidario Colombia, 2015).

Los reconocimientos mostrados anteriormente son muestra de que las universidades en su papel de organizaciones deben responder por los impactos que generan, especialmente a la sociedad. Es así como la extensión y proyección social constituye una forma, no de realizar filantropía o asistencialismo, sino de comprender el entorno en que estas instituciones se encuentran inmersas y de vincularse plenamente con la comunidad. En este caso, la extensión se ofrece como forma de conducir impactos sociales que genera la Universidad. La extensión y proyección social es una función misional de las universidades que permite la reciprocidad entre academia y conocimiento y las necesidades de la sociedad.

\section{Conclusiones}

Es indudable que la educación superior requiere del liderazgo asertivo de multiplicidad de actores que en este contexto corresponde al Estado, la universidad y las empresas. Precisamente Hurtado (2014), en un destacado estudio sobre los modelos interpretativos de la relación estado-empresa-universidad, asegura que la interacción entre estos tres actores es la "respuesta a los actuales escenarios competitivos en donde el conocimiento es fuente generadora de nuevas estructuras productivas y económicas al ser utilizado como combustible para el desarrollo de una sociedad" (p. 112).

Así, resulta también de gran interés y utilidad atender a lo expuesto por Salazar, Suescún, y Cuentas (2013), quienes realizan un interesante 
aporte sobre la Cooperación Universidad Empresa (CUE) en el estudio denominado "Factores organizativos determinantes de éxito en la cooperación universidad-empresa: un modelo integrado". Salazar, Suescún y Cuentas (2013) destacan que la universidad como organización juega un importante papel en el éxito de la explotación del conocimiento, el cual está determinado por las estructuras, los sistemas y políticas, la orientación estratégica, las habilidades de gestión y la cultura.

En este orden de ideas, las universidades, como promotoras de uno de los servicios más importantes para la sociedad, enfrentan el gran reto de orientar su filosofía de gestión y liderazgo hacia modelos más innovadores e incluyentes que no solo propicien el establecimiento de relaciones estratégicas con su entorno más inmediato, es decir, la comunidad que le rodea, sino que también promuevan el bienestar general de grupos de interés internos como docentes, estudiantes, personal de apoyo y egresados, quienes integran la comunidad universitaria y hacen posible el ejercicio de las funciones misionales de las instituciones de educación superior: docencia, investigación y extensión y proyección social. 


\section{Referencias}

Barroso, F. (2009). Responsabilidad social empresarial y sugerencias para su aplicación en instituciones educativas. Barranquilla: Universidad Simón Bolívar.

Beltrán, J., Íñigo, E. y Mata, A. (2014). La responsabilidad social universitaria, el reto de su construcción permanente. Revista Iberoamericana de Educación Superior, 14, 3-18.

Bernal, H. y Rivera, B. (2011a). Responsabilidad Social Universitaria. Aportes para el análisis de un concepto. En Asociación colombiana de Universidades, Ascún, Responsabilidad Social Universitaria. Bogotá, D.C.: Editorial Kimpres Ltda.

Bernal, H. y Rivera, B. (2011b). Responsabilidad Social Universitaria. Pensamiento Universitario, 21, 7-19. Asociación Colombiana de Universidades.

Caira, N., Lescher, M. y Lescher, I. (2015). Responsabilidad social universitaria en Maracaibo, Venezuela. Telos. Revista de Estudios Interdisciplinaros en Ciencias Aociales, 17, 196-207.

Campbell, J. L. (2007). Why would corporations behave in socially responsible ways? An institutional theory of corporate social responsibility. Academy of Management Review, 32(3), 946-967.

Cemefi. (s.f.). Centro Mexicano para la Filantropía. Recuperado de http:// www.cemefi.org/esr/

Corporación Fenalco Solidario Colombia. (2015). Informe de Gestión 2015. Recuperado de http://www.fenalcosolidario.com/ informacion-corporativa\#quienesSomos

Dacin, M. T., J. Goodstein and W. R. Scott. (2002). Institutional theory and institutional change: Introduction to the Special Research Forum. Academy of Management Journal, 45(1), 45-57.

Dulzaides, M. E., y Molina, A. M. (2004). Análisis documental y de información: dos componentes de un mismo proceso. Acimed, 12(2), 1-1.

Domínguez, M. (2009). Humanismo y trabajo social. Recuperado de https://buleria.unileon.es/bitstream/handle/10612/1498/Hum8_art1.pdf?sequence=1

Domínguez, M. J. (2012). Responsabilidad Social Universitaria. Recuperado de https://buleria.unileon.es/bitstream/handle/10612/1498/Hum8_art1. pdf? sequence $=1$ 
Global Compact. United Nations. The Ten Principles of the un Global Compact. Recuperado de https://www.unglobalcompact.org/what-is-gc/ mission/principles

Grondona, G. y Rodríguez, M. (2000). Universidad y sociedad: análisis de una experiencia de psicología social comunitaria en Quito. Ecuador: Ediciones Abya-Yala.

Herrera, G. M. y Jaime, C. A. (2004). Generación y transformación de las instituciones sociales: los procesos morfoestáticos y los procesos morfogenéticos. Reis, 107(4), 49-87.

Hurtado, J. L. (2014). Modelos interpretativos de la relación estado-empresa-universidad. Clío América, 8(15), 111-122.

Mendoza-Fernández, D. L., Salas-Solano, E. y López-Juvinao, D. D. (2015). Responsabilidad social en universidades públicas autónomas: Esencia académica con visión transformadora. Clío América, 9(18), 135-142.

Ojalvo, V. y González, B. (2014). La responsabilidad social universitaria para la formación. La Habana: Editorial Universitaria.

North, D. C. (1991). Institutions, Institutional Change and Economic. Perspectives, 5(1), 97-112.

Rodríguez, E. (2009). El rol de las universidades en la sociedad del conocimiento y en la era de la globalización: evidencia desde Chile. Asociación Interciencia Venezuela, 34(11), 822-829.

Ruiz, J. (2015). Responsabilidad Social Empresarial Universitaria: una responsabilidad interna. Cuadernos Latinoamericanos de Administración, XII(21), 5-7.

Salazar, P. V., Suescun, E. A., y Cuentas, G. A. (2013). Factores organizativos determinantes de éxito en la cooperación universidad-empresa: un modelo integrado. ALTEC.

Universidad del Magdalena (Groncallo). (21 de mayo de 2015). Unimagdalena. Obtenido de http://www.unimagdalena.edu.co/Lists/Noticias/ DispForm.aspx?ID=1652\&ContentTypeId=0x0100DFC0AB27DAE9E74F8F101938380C5DAE

Universidad del Magdalena (s.f.). Informe de Gestión Vicerrectoría de Extensión y Proyección Social 2012-2016. 100 logros de impacto en el desarrollo social y cultural desde la extensión universitaria. Universidad del Magdalena, Colombia. 
Universidad del Magdalena. Vicerrectoría de Extensión y Proyección Social. (s.f.). Presencia Institucional de la Universidad del Magdalena. Recuperado de http://extension.unimagdalena.edu.co/extension/Paginas/PresenciaInstitucional.aspx

Universidad del Magdalena. Vicerrectoría de Extensión y Proyección Social. (2015). Modalidades de Extensión. Recuperado de http://extension.unimagdalena.edu.co/extension/Paginas/ModalidadesdeExtensi\%C3\%B3n. aspx

Vallaeys, F., De la Cruz, C. y Sasia, P. M. (2009). Responsabilidad Social Universitaria. Manual de primeros pasos. México, D.F.: McGraw Hill.

Vallaeys, Fr. (2006). Breve marco teórico de responsabilidad social universitaria. Recuperado de http://rsuniversitaria.org/web/images/stories/BreveMarcoTeodelaResponsabilidadSocialUniv.pdf

Vallaeys, F. (2014). La responsabilidad social universitaria: un nuevo modelo universitario contra la mercantilización. Revista Iberoamericana de Educación Superior, 5(12), 105-117. 